\title{
Three atypical manifestations of granulomatosis with polyangiitis: lateral medullary syndrome, anterior cheek mass and melting scleritis of eye
}

\author{
Saeedeh Shenavandeh ${ }^{1}$, Peyman Petramfar ${ }^{2}$ \\ ${ }^{1}$ Shiraz University of Medical Sciences, Departments of Internal Medicine, Division of Rheumatology, Iran \\ ${ }^{2}$ Clinical Neurology Research Center, Departments of Neurology, Shiraz University of Medical Sciences, Iran
}

\begin{abstract}
Granulomatosis with polyangiitis (GPA, formerly Wegener granulomatosis) is a vasculitis with various organ involvement. There have been a few cases of CNS stroke and rare cases of lateral medullary infarction (LMI) as a manifestation of GPA. Also there have been reports of sinuses, nose and laryngeal masses mistakenly referred as carcinomas and subsequently GPA was diagnosed in their pathological reports. Another severe fulminant manifestation can be necrotizing scleritis leading to perforation of sclera. Therefore, here we present some rare and fulminant manifestations of GPA in 3 separate cases for further emphasis of the unusual manifestations of GPA that should always be kept in mind.
\end{abstract}

Key words: granulomatosis with polyangiitis, lateral medullary infarction, scleritis, facial mass, facial nerve palsy.

\section{Introduction}

Granulomatosis with polyangiitis (GPA, formerly Wegener granulomatosis) is a vasculitis with various organ involvement, mostly upper and lower respiratory tract and kidney [1]. Unusual involvement of other organs has been reported before. We here report 3 unusual first presentations of GPA where after these manifestation other symptoms and signs progressively developed. The first case concerns a man with lateral medullary infarction (LMI) (also called Wallenberg syndrome) then pulmonary hemorrhage and proteinuria. The second case concerns a diabetic woman who presented with carotid artery stenosis and then a left cheek mass along with osteomyelitis of the maxillary bone. The third case concerns a woman with severe melting scleritis.

\section{Case presentation}

\section{Case 1}

A 42-year-old man presented with 1.5 months of dysphagia, mild headache and vertigo treated as depression.
From 1 week prior to admission the patient also developed fever, hoarseness, diplopia, urinary incontinence and imbalance, which made him completely bedridden and unable to eat, so he was referred to the emergency ward of internal medicine. On arrival the patient had fever, anisocoria (Horner's syndrome), vertical and horizontal nystagmus, numbness of the left side of the face (territory of trigeminal nerve), impaired gag reflex, wide base ataxic gate and unilateral dysmetria, so he was transferred to the neurology ward. In brain MRI studies acute ischemic infarction on the left side of the medulla, pan sinusitis, bilateral mastoiditis and a few old ischemic spots in the white matter of cerebral hemispheres were observed, so lateral medullary infarction (LMI) (also called Wallenberg syndrome) was diagnosed (Fig. $1 \mathrm{~A}-\mathrm{C}$ ) and the patient was treated with acetylsalicylic acid (ASA) and clopidogrel as a young age CVA. Therefore CVA work-ups including vasculitis work-up, viral markers, heart, biochemistry, and cerebral spinal fluid evaluation were requested. CT angiography of cervical and brain arteries including carotid and vertebral arteries and transesophageal echocardiography 

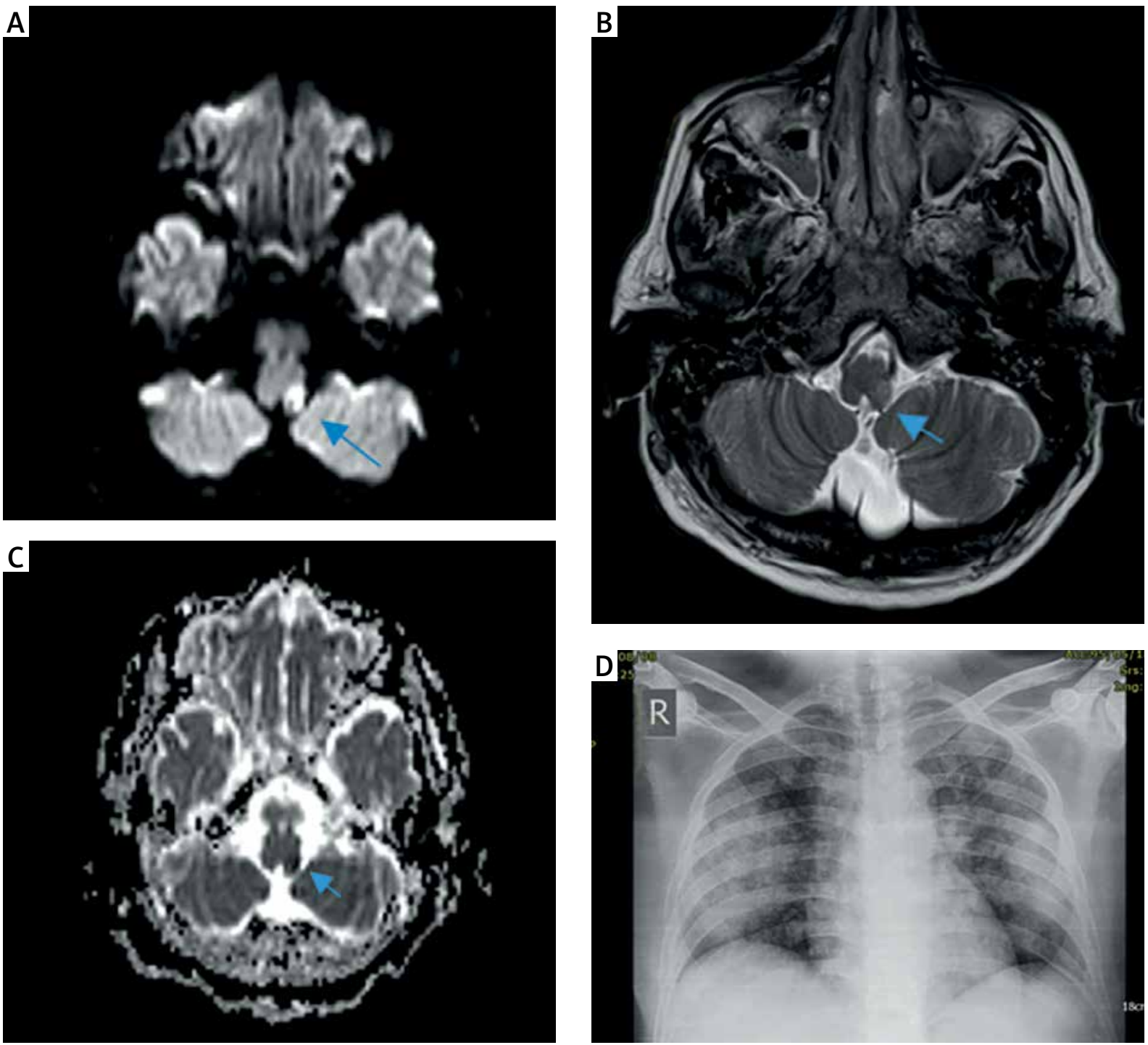

Fig. 1. A) Abnormal signal intensity in the left side of medulla is noted with diffusion restriction (DWI) MRI suggestive of acute ischemic infarction in the left side of the medulla. B) Brain MRI without contrast shows hyper signal change in lateral medulla. C) Abnormal signal intensity in the left side of medulla with ADC map of MRI suggestive of acute ischemic infarction in the left side of the medulla. D) Chest X-ray after $20^{\text {th }}$ day of admission showing diffuse bilateral lung hemorrhages.

were normal. Due to fever antibiotic for sinusitis was started. However, after negative pan-cultures due to high ESR (96) the patient was transferred to the rheumatologic ward for further evaluations. On admission the patient had fever (after 10 days of antibiotic treatment for pan-sinusitis), mild diplopia and weak gag reflex using the nasogastric tube for eating. Lung examination was normal. The patient still had urinary incontinence requiring use of an external Foley catheter. The eye assessment showed left eye pan-uveitis and in the right eye peripheral retinal vasculitis. Lung CT scan showed a focal zone of the fibrotic band and traction bronchiectasis in the posterior aspect of the right lower lobe suggestive of aspiration pneumonia. No pulmonary nodule or cavity formation was seen. In the patient serum cytoplasmic antineutrophil cytoplasmic antibodies were present (c-ANCA) and he had daily proteinuria - $750 \mathrm{mg} /$ day. The sinus biopsy showed presence of necrotic tissue with severe acute inflammation. Due to multiple organ involvement, especially CVA, proteinuria, retinal vasculitis and pan-uveitis, treatment with methylprednisolone intravenous pulses (consecutive 3 days) followed by oral prednisolone $60 \mathrm{mg} /$ day and oral cyclophosphamide $150 \mathrm{mg}$ per day was started. After 1 week the fever subsided and the patient gradually tolerated food orally. Also the patient could control his urination. 
Table I. Laboratory tests of 3 patients on arrival and during hospital coarse

\begin{tabular}{|c|c|c|c|c|c|c|c|c|c|c|}
\hline $\begin{array}{l}\text { Patients/ } \\
\text { lab } \\
\text { tests* }\end{array}$ & $\begin{array}{l}\text { WBC per } \\
\text { microliter } \\
\text { (mcl) }\end{array}$ & $\begin{array}{l}\mathrm{Hb}^{*} \\
(\mathrm{~g} / \mathrm{dl})\end{array}$ & $\begin{array}{l}\text { Platelet } \\
\text { (per } \\
\text { microliter) }\end{array}$ & $\begin{array}{c}\text { BUN } \\
\text { (mg/dl) }\end{array}$ & $\begin{array}{l}\text { Creati- } \\
\text { nine } \\
\text { (mg/dl) }\end{array}$ & $\begin{array}{c}\text { ESR } \\
(\mathrm{mm} / \mathrm{hr})\end{array}$ & $\begin{array}{l}\text { CRP } \\
(\mathrm{mg} / \mathrm{l})\end{array}$ & $\begin{array}{c}\text { Antiproteinase } 3 \\
\text { antibodies }\end{array}$ & $\begin{array}{c}\text { Urine } \\
\text { analysis } \\
\text { (U/A) }\end{array}$ & $\begin{array}{l}24 \text { hour urine } \\
\text { protein }\end{array}$ \\
\hline Case 1 & 15800 & $13.2-8.5$ & 199000 & $22-31$ & $0.9-1.4$ & $96-83$ & 192-96 & $\begin{array}{c}8.7 \\
\text { (normal: } \\
<0.4 \mu / \mathrm{ml} \text { ) }\end{array}$ & $\begin{array}{l}\text { 8-10 WBC, } \\
\text { 2-4 RBC, } \\
\text { trace albu- } \\
\text { min, 3-4 } \\
\text { granular } \\
\text { cast }\end{array}$ & $\begin{array}{l}270 \text { mg/day - } \\
490 \text { mg/day }\end{array}$ \\
\hline Case 2 & 8600 & 12.6 & 231000 & $16-22$ & $1.1-1.3$ & $68-59$ & negative & $\begin{array}{c}0.1 \text { (negative) } \\
\text { (normal: < } 0.5 \text { ) }\end{array}$ & normal & 120 mg/day \\
\hline Case 3 & 11600 & $11.3-13$ & 29450 & 15 & 0.8 & 89 & 96 & $\begin{array}{c}69 \\
\text { (normal: }<0.5)\end{array}$ & $\begin{array}{c}2+ \\
\text { albumin, } \\
\text { 3+ blood, } \\
\text { 30-35 RBC }\end{array}$ & 440 mg/day \\
\hline
\end{tabular}

\section{*Hemoglobin}

There was a history of 2 days of cough and mild bloody sputum, but the clinical assessment of the lung remained normal. Control chest X-ray (Fig. 1D) showed bilateral pulmonary hemorrhage so 7 days of plasmapheresis was started. After this course of treatment the patient was discharged in a good condition. In follow-up after 2 months the patient had no fever, could eat normally and had normal urinary continence, but still felt imbalance and severe unilateral head and facial pain. There was no proteinuria in the daily collection of urine so the treatment was continued. Lab tests during the hospital course are shown in Table I.

\section{Case 2}

A 54-year-old woman with diabetes mellitus (DM) type 2 and hyperlipidemia controlled on long-acting insulin, metformin and atorvastatin was admitted to the neurology ward with symptoms of acute headache, dip- lopia, vertigo and vomiting. In physical examination she had left-sided peripheral facial nerve palsy, and right homonymous hemianopia.

Attention was paid to accelerated ESR - 68/hour. The MRI study of the brain revealed tiny subacute ischemic spot in the right frontal lobe, intracranial right internal carotid artery (ICA) stenosis, mild pan-sinusitis (Fig. 2A) and right side mastoiditis. Color Doppler sonography of both carotid arteries also showed total occlusion of the right internal carotid artery. Sinus CT scan showed mild mucosal thickening in all paranasal sinuses suggestive for pan-sinusitis. Due to headache lumbar puncture was done and showed 40 leucocytes, 97\% lymphocytes and normal picture of cytology, and negative culture. A temporal artery biopsy was also performed and no abnormalities were observed. The patient was discharged with antibiotics with the diagnosis of impression of sinusitis, facial nerve palsy,
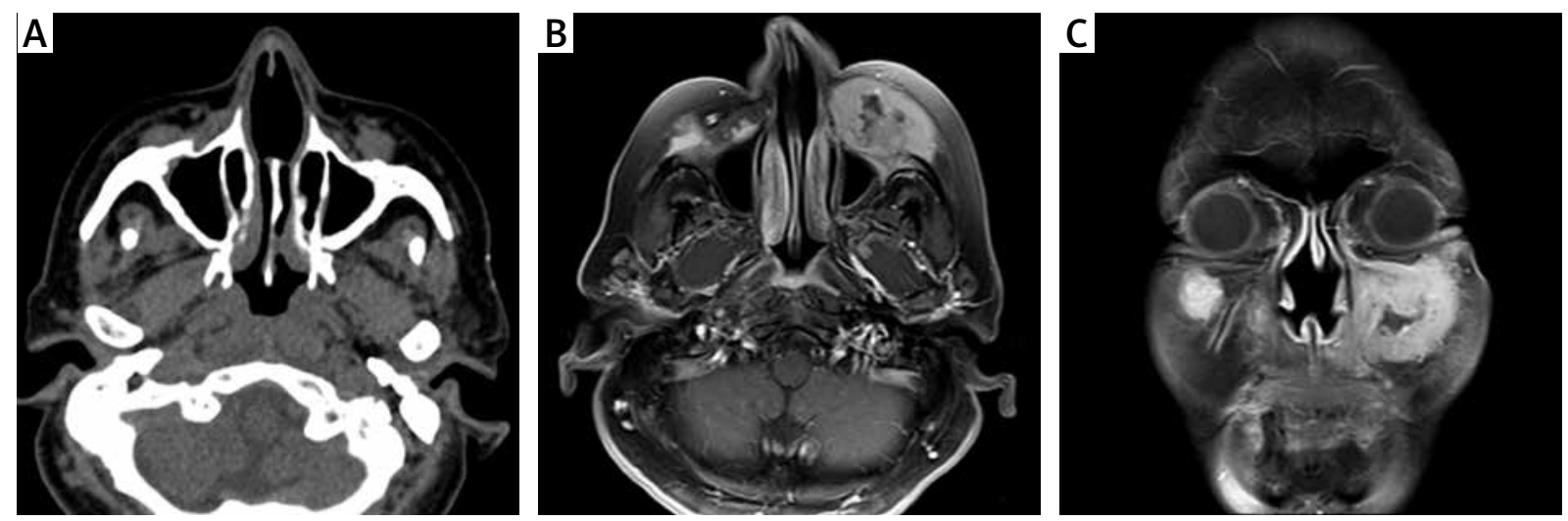

Fig. 2. A) Sinus CT Scan showing mild pan-sinusitis 6 months before last presentation. B and C) MRI of sinuses after 6 months shows destruction of anterior wall of left maxillary sinus accompanied with a large soft tissue component showing enhancement following contrast administration suggesting osteomyelitis. In the right side also osteomyelitis is noted with smaller size bone destruction and soft tissue component. 
meningitis and right internal carotid artery thrombosis. The patient gradually developed left cheek swelling and protrusion without any fever and after 5 months needed aspiration of the left maxillary sinus and histopathological assessment showed necrotizing granulomatous inflammation of the sinus. So the patient was admitted to the rheumatology ward. On admission the patient interview revealed 5 months history of bilateral hearing loss, mild left side peripheral facial nerve palsy and a protruded left cheek mass, non-tender and hard on examination. Other examinations were unremarkable. Still high ESR was observed (lab tests in Table I). MRI of the left maxillary sinus showed destruction of the anterior wall of the left maxillary sinus accompanied by a large soft tissue component showing enhancement following contrast administration suggesting osteomyelitis. On the right side also osteomyelitis was noted with smaller bone destruction and soft tissue component. Mucosal thickening of sphenoid and ethmoid air cells was also noted (Fig. 2B, C). An open sinus biopsy also showed ulceration with granulomatous tissue formation, thrombosed vessels and some eosinophilic infiltrations suggesting granulomatosis with polyangiitis (GPA). The PCR screening for tuberculosis was negative. The patient had negative ANCA, no eosinophilia and normal lung CT scan and also no kidney involvement. Due to severe involvement of sinuses and hearing loss treatment with prednisolone and oral cyclophosphamide was started and in the follow-up after 2.5 months showed complete resolution of the cheek mass.

\section{Case 3}

A 48-year-old woman with a history of polyarticular arthritis 3 years ago with high ESR and CRP and nega-

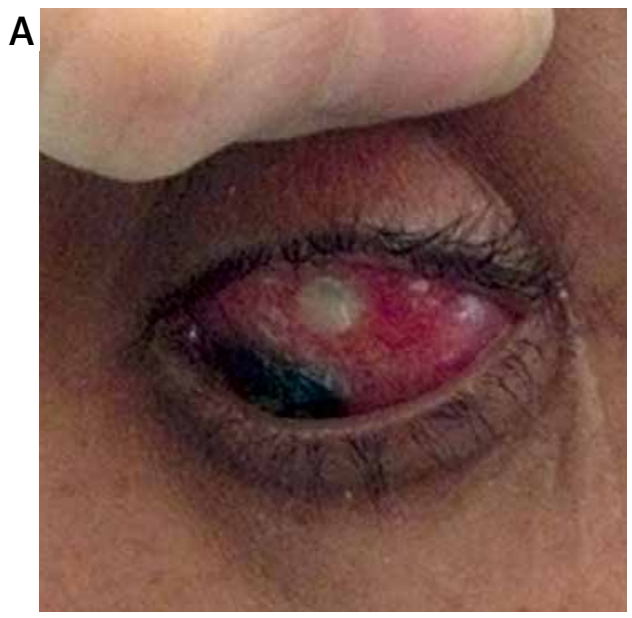

Fig. 3. A) Melting necrotizing scleral ulcer before treatment. B) 4 months after receiving treatment and scleral graft tive rheumatoid factor and anti-cyclic citrullinated peptide (ACPA) was treated for early arthritis with prednisolone and methotrexate but after the second visit due to a good response the patient discontinued all medications and did not show up for the next follow-up visits. In subsequent years the patient had some arthralgia, after that using only NSAIDs.

A month prior to admission she developed left eye pain and photosensitivity so the patient was referred to ophthalmologists and received some topical treatment without any good response. Gradually the pain increased and the patient developed blurred vision and a pussy discharge from the eye. So with the impression of severe left eye scleral melting and necrosis and with diagnosis of pan-uveitis the patient was referred to a rheumatology clinic for further examinations and aggressive treatment. On admission the patient had a history of one week of increased arthralgia, productive cough and dyspnea at night not responding to oral antibiotics. On examination the patient had a temperature of $37.9^{\circ} \mathrm{C}$ (oral measurement), chemosis and ulceration with pussy discharge of the left eye (Fig. 3A), normal lung examination and bilateral MCP and PIP signs of arthritis. Chest CT scan revealed multiple cavity formations. Serum showed c-ANCA antibodies and daily proteinuria was found (440 mg/day). The diagnosis of GPA was established and methylprednisolone pulse $1 \mathrm{gram}$ for three consecutive days was started and also cyclophosphamide (intravenous route) every two weeks for 3 doses and then every 3 weeks was followed in the OPD clinic of rheumatology and ophthalmology.

After 2 months the patient had mild improvement of data but still there was activity of eye involvement and the patient was scheduled for a scleral graft. So we decided on treatment with rituximab (intravenous infusion 1 gram, repeated after 15 days). After 5 months the

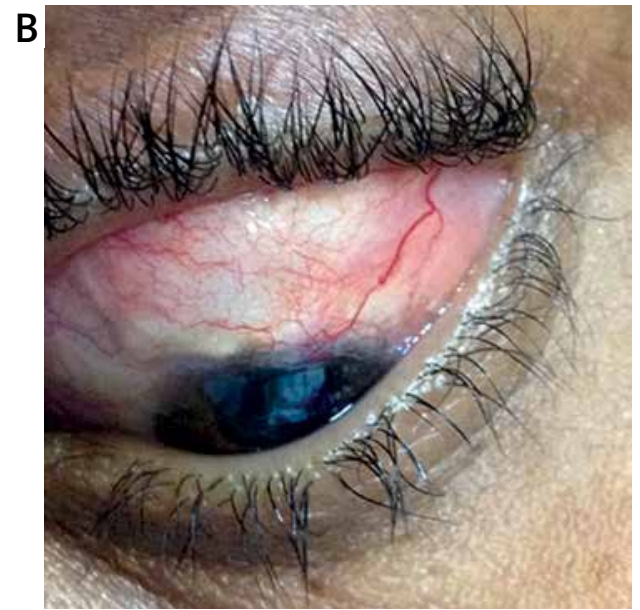


patient was treated with azathioprine $50 \mathrm{mg}$ per day and $7.5 \mathrm{mg}$ prednisolone with good response (Fig. 3B) and at the $6^{\text {th }}$ month after the first cycle of rituximab the patient because of flare of pan-uveitis received the second dose of rituximab and remains with a good response.

\section{Discussion}

We reported three rare and odd manifestations of GPA. In the first one, the patient presented with LMI of the brain and then rapidly showed other manifestations of GPA including pan-sinusitis, mastoiditis, proteinuria and lung hemorrhages. LMI is caused by a vascular event in the territory of the posterior inferior cerebellar artery or the vertebral artery. It can present with sensory deficits affecting the extremities and the trunk on the contralateral side and the face on the ipsilateral side of the infarction, along with vertigo, dysarthria, dysphagia, ataxia, ipsilateral paralysis of the soft palate, larynx, and pharynx and Horner syndrome. Headache and vertigo are the main symptoms [2]. There have been a few associations of LMI and vasculitis reported before, such as a case report including Behçet's disease [3], giant cell arteritis [4] and relapsing polychondritis [5]. Involvement of the nervous system in GPA is frequently reported (in $22-54 \%$ of cases), most commonly manifesting as peripheral neuropathy (in $10.6-21.2 \%$ of patients) or mononeuritis multiplex. Cerebral and meningeal involvement are uncommon, however, occurring in only $2-8 \%$ of patients [6]. Ischemic stroke is a rare event and usually presents in the acute phase of untreated GPA [7]. The most important mechanisms are vasculitis of the medium-sized intracranial arteries with associated thrombosis, cardiac embolism, arterial dissection, and direct invasion from the nasal cavity [8].

There have been two cases of LMI to date as a manifestation of GPA: the first one was presented by Faust et al., who reported LMI in a case of GPA with marked involvement of the lungs and upper airways but only minimal kidney disease and detected c-ANCA with the target antigen proteinase 3 (PR 3) in the cerebrospinal fluid (CSF) [9]. The second one was by Taraschenko et al. [8], who reported a 41-year-old man with lateral medullary infarction who rapidly developed progressive renal failure after that. He was diagnosed with GPA based on positive serum C-ANCA and pauci-immune crescentic glomerulonephritis on kidney biopsy. In our case other severe manifestations progressed after this brain infarction so GPA should be considered in differential diagnosis of stroke in young patients.

The second case was a woman with DM who presented with high ESR, headache, facial nerve palsy, lym- phocytic meningitis, internal carotid artery thrombosis and mild sinusitis, and who gradually developed bilateral peripheral hearing loss, sinusitis and a facial soft tissue mass invading the maxillary sinus with the pattern of osteomyelitis.

In GPA cranial nerve palsy may occur as single or multiple cranial nerve involvement and may precede the involvement of other organs. Palsies of cranial nerves (especially the third, fifth, sixth, seventh and eighth) are present in around 50\% of pachymeningitis cases [10]. Nagashima et al. [11] also reported a patient with occlusion of the ophthalmic artery and narrowing of the ICA, hypertrophic pachymeningitis and multiple cranial neuropathies as a presentation of GPA.

There have also been reports of GPA with ENT masses in the sinus, ear and nose first referred as a carcinoma $[12,13]$. Our patient did not have positive ANCA, lung and kidney involvement so the main diagnosis was the 'limited' or 'localized' form of GPA. In differential diagnoses we considered EPGA (eosinophilic granulomatosis with polyangiitis) but the tissue infiltration of eosinophils was limited and she did not have peripheral eosinophilia, asthma, peripheral neuropathy or cardiomyopathy. Also involvement of the upper airway is rare in patients with EPGA. So it seems we should always be concerned about GPA in patients of any range of age presenting with facial nerve palsy and facial masses.

The third patient presented with polyarthritis for 3 years that in the first visit was managed as early arthritis with no presence of anti-CCP antibodies or RF but did not have any follow-up for the next 3 years and there was no joint destruction despite lack of proper treatment. However, it is known that involvement of sclera is possible in rheumatoid arthritis, but at the same time the patient had cavitary lung lesions and kidney involvement and presence of c-ANCA antibodies, which is a picture suggestive for GPA. Eye involvement was seen in up to $58 \%$ of patients with GPA. The most common manifestations are orbital disease, scleral/episcleral involvement (3.5\%) and corneal and nasolacrimal abnormalities [14]. In some reports scleral involvement was higher. Hoffman et al. [1] reported that scleritis was the third most common ocular manifestation in patients with GPA. Our patient had a very good response to rituximab treatment and graft for ulcer. There have been previous reports of using surgery [15] and rituximab in refractory scleritis before [16].

Thus, it seems that GPA should always be considered in evaluations for scleritis and it may exhibit very fulminant progression without rapid treatment. Rituximab seems to be a good choice in cases of GPA with refractory eye manifestations. 


\section{Conclusions}

The goal of presentation of these three cases was to present some rare and unusual manifestations of granulomatosis with polyangiitis presenting with LMI, meningitis, cranial nerve palsy, facial mass and osteomyelitis, and severe scleritis to help in early diagnosis of this disease and prevent its progression.

The authors declare no conflict of interest.

\section{References}

1. Hoffman GS, Kerr GS, Leavitt RY, et al. Wegener granulomatosis: an analysis of 158 patients. Ann Int Med 1992; 116 488-498.

2. Sacco RL, Freddo L, Bello JA, et al. Wallenberg's lateral medullary syndrome. Clinical-magnetic resonance imaging correlations. Archiv Neurol 1993; 50: 609-614.

3. Gan J, Zheng HB, Xi J, et al. A case of neuro-vasculo-Behcet disease. Europ J Neurol 2007; 14: e16-17.

4. Shanahan EM, Hutchinson M, Hanley SD, Bresnihan B. Giant cell arteritis presenting as lateral medullary syndrome. Rheumatology (Oxford) 1999; 38: 188-189.

5. Topalkara K, Kaptanoglu E, Akyuz A, et al. Relapsing polychondritis with involvement of posterior inferior cerebellar artery causing acute lateral medullary syndrome. J Clin Rheumatol 2003; 9: 92-95.

6. Murphy JM, Gomez-Anson B, Gillard JH, et al. Wegener granulomatosis: MR imaging findings in brain and meninges. Radiology 1999; 213: 794-799.

7. Bares M, Muchova M, Dufek M, et al. Wegener's granulomatosis: ischemic stroke as the first clinical manifestation (case study). J Neurol 2002; 249: 1593-1594.
8. Taraschenko OD, Amory CF, Waldman J, et al. Lateral medullary stroke in patient with granulomatous polyangiitis. J Stroke Cerebrovasc Dis 2014; 23: 1259-1261.

9. Faust J, Visbeck A, Fitzek S, et al. Vasculitic wallenberg syndrome with detection of anti-proteinase 3 antibodies in the cerebrospinal fluid of a patient with severe Wegener's granulomatosis and only mild kidney involvement. Nephrol Dial Transplant 2000; 15: 893-896.

10. Holle JU, Gross WL. Neurological involvement in Wegener's granulomatosis. Curr Opin Rheumatol 2011; 23: 7-11.

11. Nagashima T, Maguchi S, Terayama Y, et al. P-ANCA-positive Wegener's granulomatosis presenting with hypertrophic pachymeningitis and multiple cranial neuropathies: Case report and review of literature. Neuropathology 2000; 1: 23-30.

12. La Rosa C, Emmanuele C, Tranchina MG, et al. Diagnostic consideration for sinonasal Wegener's granulomatosis clinically mistaken for carcinoma. Case Rep Otolaryngol 2013; 2013: 839451.

13. Yanamoto S, Kawasaki G, Yoshida H, et al. Rapidly growing mass of the anterior maxillary gingiva. Oral Surg Oral Med Oral Pathol Oral Radiol Endod 2007; 104: 153-159.

14. Tarabishy AB, Schulte M, Papaliodis GN, Hoffman GS. Wegener's granulomatosis: clinical manifestations, differential diagnosis, and management of ocular and systemic disease. Surv Ophthalmol 2010; 55: 429-444.

15. Lu CW, Zhou DD, Wang J, Hao JL. Surgical treatment of peripheral ulcerative keratitis and necrotizing scleritis in granulomatosis with polyangiitis. Saudi Med J 2016; 37: 205-207.

16. Recillas-Gispert C, Serna-Ojeda JC, Flores-Suarez LF. Rituximab in the treatment of refractory scleritis in patients with granulomatosis with polyangiitis (Wegener's). Graefes Arch Clin Exp Ophthalmol 2015; 253: 2279-2284. 